\title{
Novice Surgeon Portal Preference to Visualize the Femoral Anterior Cruciate Ligament Footprint: The Accessory Medial Portal Offers Improved Visualization
}

\author{
Mehmet Burtaç Eren, M.D., and Erkal Bilgiç, M.D.
}

\begin{abstract}
Purpose: To evaluate participant opinions on the appropriateness of different viewing angles by asking 8 questions, using visual content techniques. Methods: Survey information from participants was used in this study. We used images of a patient who was operated on for symptomatic knee instability. Participants were asked whether the visual angle could determine the femoral tunnel entry point or evaluate anterior cruciate ligament (ACL) integrity. Results: Questionnaires were completed by 40 participants. When all responses were evaluated, participant opinions on the accessory medial portal (AMP) were more positive than opinions on images taken from the anterolateral portal (ALP). These observations were statistically significant $(P<.005)$. Both ACL integrity assessments and femoral tunnel entry site questions were evaluated, we observed that participant opinions on the AMP for both types of evaluation were more positive than opinions on the ALP. This observation was statistically significant $(P<.005)$. When evaluations with photos and video transcripts were compared, no significant differences in terms of participant opinions were observed $(P<.005)$. Conclusions: AMP use may be a valuable tool for assistant and postgraduate education, as it offers a more suitable view angle for ACL reconstruction. Level of evidence: Level V, survey study.
\end{abstract}

D ifferent imaging and portal combinations serve different needs during arthroscopic surgery, where portal selection is defined according to the display area and the procedure. In recent years, major developments and changes have been implemented in arthroscopic surgery training. Computer simulations and models that provide feedback, suture stations, and laboratories have become widespread training approaches. Both in vivo and in vitro methods have been described for arthroscopic training in the knee, ${ }^{1-6}$ shoulder, ${ }^{7-9}$ ankle, $^{10}$ hip, ${ }^{11}$ and wrist. ${ }^{12,13}$ Advanced

From the Department of Orthopaedics and Traumatology, Gaziosmanpasa University School of Medicine, Tokat, Turkey.

The authors report that they have no conflicts of interest in the authorship and publication of this article. Full ICMJE author disclosure forms are available for this article online, as supplementary material.

Received July 30, 2020; accepted December 29, 2020.

Address correspondence to Dr. Mehmet Burtaç Eren, M.D., Department of Orthopaedics and Traumatology, Gaziosmanpasa University, Kaleardı District Muhittin Fisunoglu St., 60100 Tokat,Turkey.E-mail:mehmetburtac@ hotmail.com

(C) 2021 THE AUTHORS. Published by Elsevier Inc. on behalf of the Arthroscopy Association of North America. This is an open access article under the CC BY-NC-ND license (http://creativecommons.org/licenses/by-nc-nd/4.0/). 2666-061X/201119

https://doi.org/10.1016/j.asmr.2020.12.013 courses now focus on specific skillsets. ${ }^{14,15}$ In addition to these developments, the simultaneous monitoring of arthroscopic screens and the re-evaluation of arthroscopy records continue to form a vital basis for education in this subject area.

The concept that education should be reformed according to surgical needs is not new. Indeed, it is 25 years since Folse stated this issue. ${ }^{16}$ In surgical clinics, education has evolved from a master-apprentice relationship to a multidimensional and technological state. The ultimate goal of a surgeon's education is to perform surgery, and although surgical methods have changed, the goals have not. Arthroscopic images are vitally important for both arthroscopic teaching and dissemination, as the area is primarily screen based. In addition to theoretical knowledge, being able to obtain and interpret arthroscopic images is a primary educational goal. In adult education, the cognitive apprenticeship method attributes mutual roles to the educator and the trainee. The model, which provide a full qualification, is generally evaluated across 5 phases. ${ }^{17-21}$ The first 3 phases, modeling, approximating, and fading, are required for the trainer to exchange ideas via video photography (Fig 1).

In operations comprising different technical difficulties, step-by-step narration and technical tricks are 


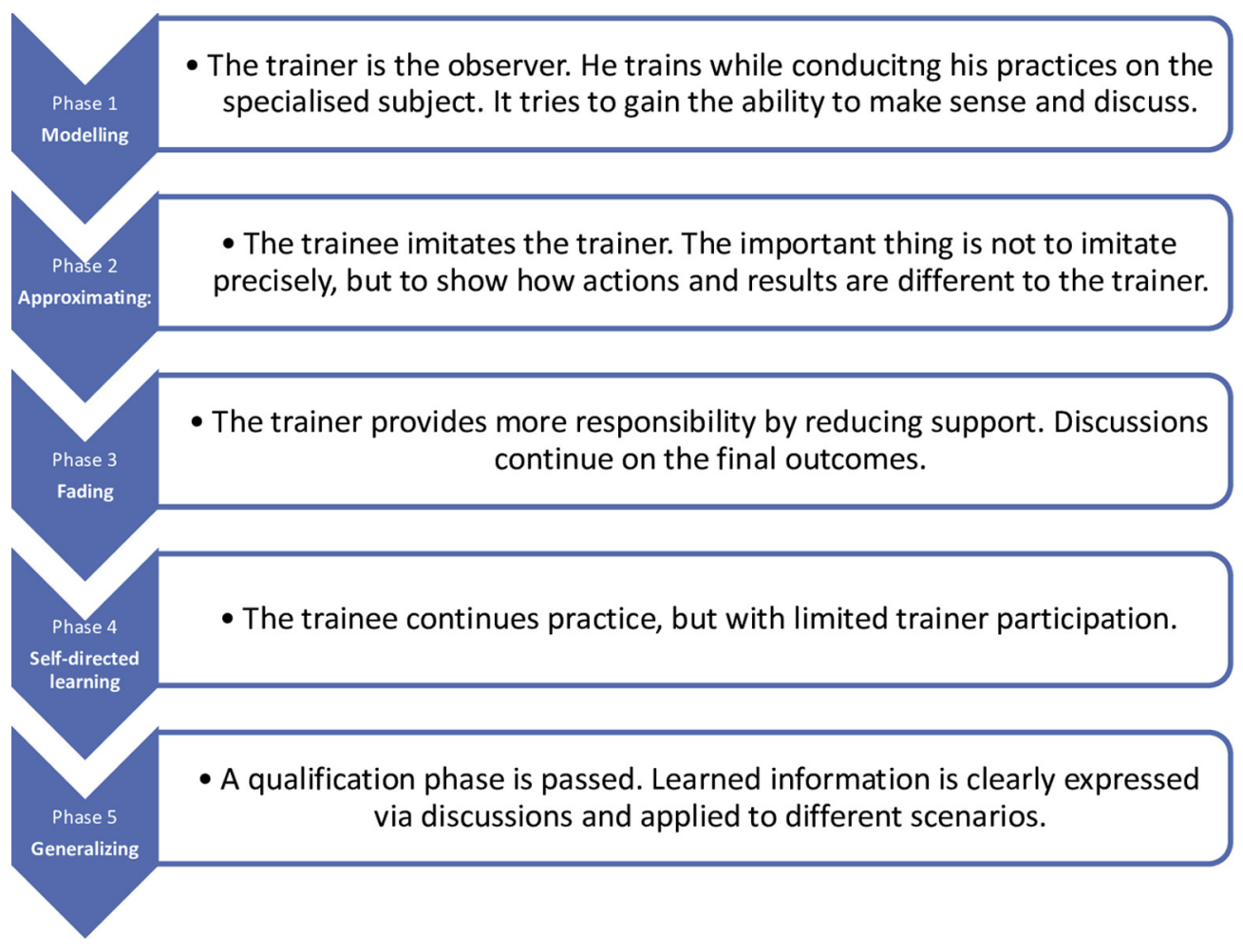

Fig 1. Cognitive apprenticeship phases.

important training points. Performance metric systems have been developed especially for specific shoulder arthroscopy procedures. These systems were created by the Delphi panel, ${ }^{22-25}$ from advanced experienced shoulder arthroscopist feedback to video recordings, and have contributed greatly to arthroscopy training. ${ }^{14,26}$ Arthroscopic video-photo recordings are greatly important for creating and implementing the metric system, which is required for the development of standard education approaches across arthroscopy. Internalizing surgical techniques, step by step, is useful preparation for forthcoming procedures.

Different technical aspects have been described for anterior cruciate ligament (ACL) reconstruction, which is a common arthroscopic procedure. Surgeon graft donor sites, graft preparations, graft fixation, bone tunnel techniques, and imaging portals all may differ. The surgeons' understanding of the techniques used in ACL reconstruction may differ. This difference may be important in terms of training novice surgeons and assistants.

Technical discussions for ACL reconstruction have been ongoing for years. Despite recent developments, there are still differences in surgical techniques. It is known that femoral tunnel placement affects clinical outcomes, ${ }^{27-30}$ and improper femoral tunnel placement is the most common cause of surgical failure. ${ }^{31}$

Many different techniques have been described in arthroscopic ACL reconstruction, and there is no common consensus for the placement of the portals. In the early years of arthroscopic ACL reconstruction, the transtibial technique became prominent for femoral tunnel opening; however, later it was avoided because it complicated horizontal tunnel placement. After this technique, the anteromedial portal technique ${ }^{32-35}$ and 3 -portal technique were defined. ${ }^{36,37}$

In all techniques, it is important to display the lateral notch wall to determine the femoral tunnel entry site. When performing arthroscopy in patients suspected of ACL damage, it is difficult to decide on essential reconstruction, especially in patients with posterior cruciate ligament adhesions. In such cases, a figure of 4 position examination is recommended. In assessing femoral footprint integrity, the lateral notch wall should be clearly displayed. ${ }^{38}$ Therefore, viewing this structure at a wide and appropriate angle is especially important for the initial evaluation of ACL integrity.

Training techniques that facilitate both lateral notch wall visualization and ACL integrity assessments may benefit assistant and postgraduate training. We aim to evaluate participant opinions on the appropriateness of different viewing angles, by asking 8 survey questions, using visual content techniques.

After evaluating answers of participant, we posed the following questions:

1. Are there significant differences in participant opinion between the 2 techniques in determining the femoral tunnel entry site? 
Fig 2. Images used in questions. (A) Screenshot 1, (B) Screenshot 2, (C) Screenshot 3, (D) Screenshot 4.
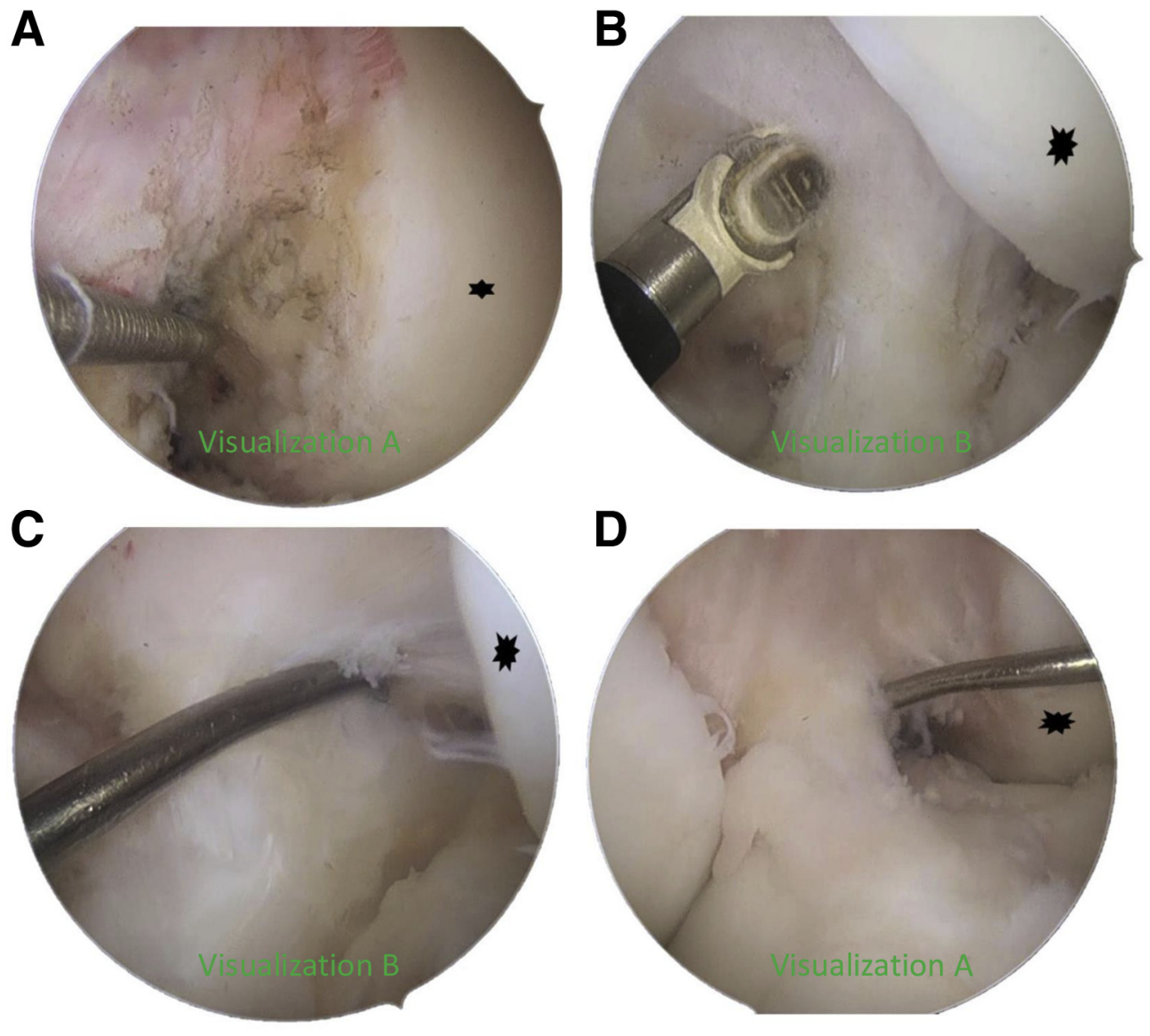

2. Are there significant differences in participant opinion between the 2 techniques in terms of ACL integrity assessment?

3. When the answers are evaluated together, are there significant differences between 2 techniques?

4. Are there differences when video sections and screenshots are compared?

We hypothesized that the images obtained from the accessory medial portal would be more acceptable for the participants' views.

\section{Methods}

Patients who had surgery for symptomatic knee instability by authors (M.B.E., E.B.) between December 2017 and December 2019 were identified through a search of our hospital information management system. Inclusion criteria were being operated with the diagnosis of isolated ACL rupture, well-documented adhesions of ruptured ACL during surgery, and being between the ages of 15 and 45 years. Exclusion criteria were patients with insufficient video recording, having concomitant knee surgery, and having a cyclops lesion on the residual ACL footprint. There were 5 of 22 patients who met the criteria. The images of the patient who had the longest arthroscopy record were used (Fig 2).

When images were acquired, Cohen et al., ${ }^{36} 3$ portal techniques were used. In addition to a standard anterolateral portal (ALP), the central medial portal was opened immediately adjacent to the patellar tendon. Then, for imaging, an accessory medial portal (AMP) was created using an 18-gauge spinal needle using the outside-in technique from $2 \mathrm{~cm}$ medial to the patellar tendon. During routine surgical procedures, imaging was performed via different portals for 5 minutes before and after footprint resection. The images obtained when evaluating the notch lateral wall and ACL integrity using AMP were processed and prepared for use in questions (visualization A).

Then, images taken from the ALP were processed before the accessory medial portal was created. Images were prepared for use in the questionnaire (visualization B).

The arthroscopy film was processed using a videoediting program, and 4 snapshots and 4 video sections, ranging from 52 to 112 seconds, were prepared. The images were added to the 8 question images to provide equal numbers of video sections and screenshots, from both ALP and AMP. 
Table 1. Question Distribution

\begin{tabular}{|c|c|c|c|}
\hline Question Number & Used Image & Viewing Portal & Criteria Evaluated \\
\hline 1 & Screenshot 1 & Visualization A & Femoral tunnel entry site determination \\
\hline 2 & Screenshot 2 & Visualization B & Femoral tunnel entry site determination \\
\hline 3 & Screenshot 3 & Visualization B & ACL integrity assessment \\
\hline 5 & Video 1 & Visualization B & ACL integrity assessment \\
\hline 6 & Video 2 & Visualization A & ACL integrity assessment \\
\hline 7 & Video 3 & Visualization B & Femoral tunnel entry site determination \\
\hline
\end{tabular}

Survey information from participants was used in this study.

All images showed the left knee from the same patient, with a $30^{\circ}$ scope and the knee at $90^{\circ}$ flexion. Questionnaires were prepared by placing an equal number of both portal techniques in the case. A case was chosen that was ruptured and adhered to posterior cruciate ligament. A questionnaire with 8 questions in total was prepared to question both techniques equally (Table 1).

Participant inclusion criteria was in the last 3 years of being orthopaedic and traumatology assistant training and having arthroscopic ACL reconstruction experience of fewer than 100 cases. Participant exclusion criteria were having orthopaedic expertise of 10 years or greater.

After the survey was prepared, orthopaedics assistants and experts who met the criteria were contacted and the survey link was shared. The survey statistics were checked daily and the questionnaire was terminated when 40 participants were reached. Participants were asked whether the visual angle could determine the femoral tunnel entry point, or evaluate ACL integrity. In the photo questions, the lateral femoral condyle was marked with an asterisk, and given as a reference point. Participants were informed the knee was in $90^{\circ}$ of flexion, and used with a $30^{\circ}$ scope.

For ACL integrity evaluation, images taken before the footprint were debrided and used, whereas images taken after debridement were used to evaluate the femoral tunnel entry site.

The femoral tunnel evaluation question was: "does the viewing angle determine the femoral tunnel entry point, where ACL reconstruction will be performed?" The ACL integrity assessment question was: "is the viewing angle suitable to ask whether the patient, who was operated on for suspicious ACL rupture, displays ACL integrity?" Answers were evaluated in 5 categories, according to the Likert scale: (1) strongly disagree; (2) disagree; (3) undecided; (4) agree; and (5) strongly agree.

The survey was conducted via Google Forms (Google, Mountain View, CA; the full survey is available online as an Appendix Supplement 1, available at www. arthroscopyjournal.org). Whereas images were

Table 2. Distribution of Response Variables

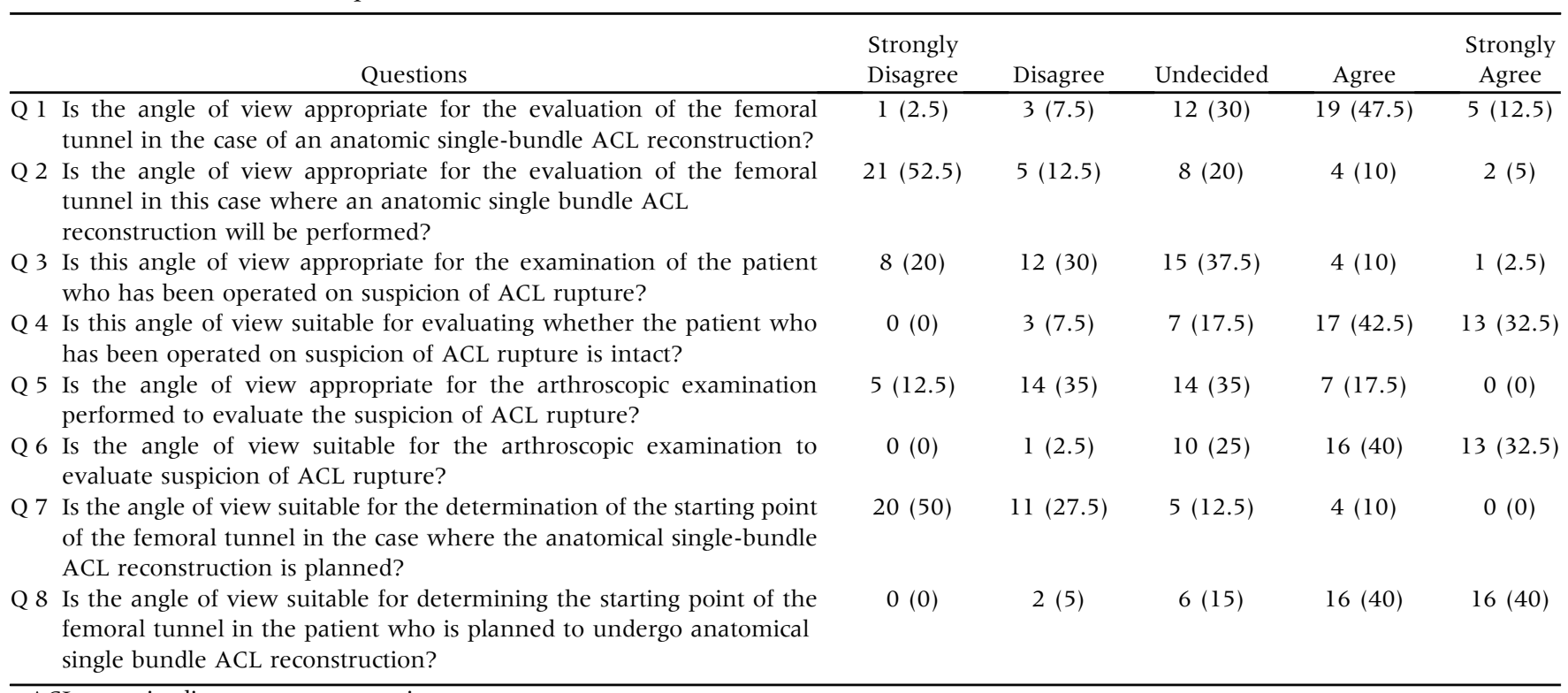

ACL, anterior ligament reconstruction. 
Table 3. Distribution of Answers by Technique

\begin{tabular}{|c|c|c|c|c|}
\hline & \multicolumn{2}{|c|}{ Technique } & \multirow[b]{3}{*}{$\chi^{2}$} & \multirow[b]{3}{*}{$P$} \\
\hline & $\begin{array}{c}\text { Visualization } \\
\mathrm{A}\end{array}$ & $\begin{array}{c}\text { Visualization } \\
\text { B }\end{array}$ & & \\
\hline Answers & $\mathrm{n}(\%)$ & n (\%) & & \\
\hline Strongly disagree & $1(0.6)^{*}$ & $54(33.8)^{\dagger}$ & 139,380 & $<.001$ \\
\hline Disagree & $9(5.6)^{*}$ & $42(26.3)^{\dagger}$ & & \\
\hline Undecided & $35(21.9)^{*}$ & $42(26.3)^{*}$ & & \\
\hline Agree & $68(42.5)^{*}$ & $19(11.9)^{\dagger}$ & & \\
\hline Strongly agree & $47(29.4)^{*}$ & $3(1.9)^{\dagger}$ & & \\
\hline
\end{tabular}

NOTE. The $\chi^{2}$ test was also used.

*The line letter is statistically insignificant in terms of expression.

${ }^{\dagger}$ The line letter is statistically insignificant in terms of expression.

included in the online survey (Fig 2), videos were uploaded to 2 different video sharing sites (Yandex Disk, Moscow, Russia; and YouTube [Google, D/B/A YouTube, San Bruno, CA]), and video links were processed into the survey form.

In terms of ACL examination and determination of femoral tunnel entry point, we evaluated whether significant difference existed between the AMP and ALP images. In addition, using images from the same portals, we evaluated whether participants observed significant differences between evaluations made by video imaging or photo images.

\section{Statistical Analysis}

Data are expressed as mean \pm standard deviation or frequency and percent. An independent sample $t$ test was used to compare the continuous normal data between groups. $\chi^{2}$ test or Yates Correction $\chi^{2}$ test was used to compare the categorical data between/among groups. $P$ value $<.05$ was considered significant. Analyses were performed using SPSS 19 (IBM SPSS Statistics 19, an IBM Co., Armonk, NY).

\section{Results}

Questionnaires were completed by 40 participants. Variable distribution was created by evaluating participant responses to questions (Table 2).
When all responses were evaluated, participant opinions on the visualization A were more positive than visualization $\mathrm{B}$. These observations were statistically significant $(P<.001)$ (Table 3). According to the visualization technique, the distribution of the answers given to the questions was compared and analyzed (Table 3).

When ACL integrity assessments and femoral tunnel entry site questions were evaluated, we observed that participant opinions on the visualization A for both types of evaluation were more positive than visualization $B$. This observation was statistically significant $(P<.001)$ (Table 4$)$.

When evaluations with photos and video transcripts were compared, no significant differences in terms of participant opinions were observed $(P=.579)$ (Table 5).

\section{Discussion}

Our study participants preferred the AMP, suggesting this modality may be more appropriate for those who wish to continue their training/learning. We also observed that in determining the entry point of the femoral tunnel, our participants significantly favored images from the AMP. Sommer et al. ${ }^{39}$ evaluated femoral tunnel entry points in patients with ACL reconstruction due to persistent knee instability and demonstrated that the most common misplacement error was anterior malposition. Similarly, Morgan et al. $^{31}$ in their Multicenter ACL Revision Study (MARS), identified technical problems in 276 of 460 revision ACL cases. In 117 cases, the failure was evaluated as femoral tunnel malposition.

Improving lateral notch wall imaging may contribute to long-term outcomes in preventing femoral tunnel malposition, leading to revision ACL surgery. Intraoperative fluoroscopic radiologic evaluations that determine clinically correct femoral entry points have also been cited in the literature. Sven et al. ${ }^{40}$ detected and corrected 34 cases that would cause erroneous placement in femoral and tibial tunnels, by using fluoroscopy in 112 ACL reconstruction cases.

Table 4. Distribution of Answers by Femoral Tunnel Entry Point and ACL Integrity Assessment

\begin{tabular}{|c|c|c|c|c|c|c|c|c|}
\hline \multirow[b]{3}{*}{ Answer } & \multicolumn{2}{|c|}{ Femoral Tunnel Entry Point } & \multirow[b]{3}{*}{$\chi^{2}$} & \multirow[b]{3}{*}{$P$ Value } & \multicolumn{2}{|c|}{ ACL Integrity Assessment } & \multirow[b]{3}{*}{$\chi^{2}$} & \multirow[b]{3}{*}{$P$ Value } \\
\hline & Visualization A & Visualization B & & & Visualization A & Visualization B & & \\
\hline & $\mathrm{n}(\%)$ & $\mathrm{n}(\%)$ & & & $\mathrm{n}(\%)$ & $\mathrm{n}(\%)$ & & \\
\hline Disagree & $5(6.3)^{*}$ & $16(20)^{\dagger}$ & & & $4(5)^{*}$ & $26(32.5)^{\dagger}$ & & \\
\hline Undecided & $18(22.5)^{*}$ & $13(16.3)^{*}$ & & & $17(21.3)^{*}$ & $29(36.3)^{\dagger}$ & & \\
\hline Agree & $35(43.8)^{*}$ & $8(10)^{\dagger}$ & & & $33(41.3)^{*}$ & $11(13.8)^{\dagger}$ & & \\
\hline
\end{tabular}

NOTE. The $\chi^{2}$ test was also used.

*The line letter is statistically insignificant.

${ }^{\dagger}$ The line letter is statistically insignificant. 
Table 5. Distribution of Responses Based on Screen Shots and Video Sections

\begin{tabular}{|c|c|c|c|c|}
\hline Answers & $\frac{\text { Screenshot }}{\mathrm{n}(\%)}$ & $\frac{\text { Video Section }}{\mathrm{n}(\%)}$ & $\chi^{2}$ & $P$ Value \\
\hline Strongly disagree & $30(18.8)$ & $25(15.6)$ & 2.873 & .579 \\
\hline Disagree & $23(14.4)$ & $28(17.5)$ & & \\
\hline Undecided & $42(26.3)$ & $35(21.9)$ & & \\
\hline Agree & $44(27.5)$ & $43(26.9)$ & & \\
\hline Strongly agree & $21(13.1)$ & $29(18.1)$ & & \\
\hline
\end{tabular}

NOTE. The $\chi^{2}$ test was used.

$* P<.005$ was significant.

Of course, our study is not designed to test the clinical effects of AMP use. However, the fact that the participants found the images taken from this portal more understandable in terms of evaluating the femoral tunnel entrance, made us think that the training materials obtained from this portal imaging could be more valuable.

While the AMP femoral tunnel is being created, it cannot be used as an imaging portal. However, it may give novice surgeons a final check-up after the entry point is gently marked.

In our study, we did not perform any clinical or cadaveric studies. Nevertheless, objectively demonstrating that the AMP provides a more appropriate view in terms of participants' opinions may be a valuable finding in terms of ACL reconstruction surgery training.

Another important result from our study was that AMP images were more appropriate for participants in evaluating ACL integrity. Siebold and $\mathrm{Fu}^{41}$ stated that isolated anteromedial bundle or posterolateral bundle tears are difficult to detect, even using magnetic resonance imaging, and emphasized that isolated reconstructions also required advanced experience. They reiterated a correct diagnosis could be arrived at arthroscopically with advanced arthroscopic experience. For this reason, the images obtained from the AMP can be helpful when presenting educational material to novice surgeons especially about ACL integrity assessment and femoral tunnel entry point conformity check

We also observed no differences between participant opinions in terms of screen shots and video sections. Ceponis et al., ${ }^{42}$ in teaching shoulder arthroscopy to surgical assistants, compared composite video models and cadaveric shoulder arthroscopy training. They suggested that composite models were at least as effective as cadaver methods. It is not possible to teach arthroscopy without video sections and screenshot support. After evaluations were made with multimedia types, i.e., video-screen imaging, we observed no significant differences between participants, suggesting that both techniques can be used for ACL reconstruction training.

\section{Limitations}

This study is not without limitations. Participants did not express their opinions using a cadaver approach or a simulator. By focusing on a very specific issue, this limitation was partially resolved by only evaluating the angle of view, ACL integrity and the femoral tunnel entry site. However, these parts that have been evaluated constitute only a few stages of ACL reconstruction and cannot represent the understandability of the entire surgical procedure.

Another limitation of our study is that we routinely chose a portal not used for viewing during tunneling. We think that this limitation is not incompatible with the main idea of the study, since we have used this portal for final control of the femoral tunnel entry site and ACL integrity evaluation instead of using it as a viewing portal while opening the femoral tunnel.

\section{Conclusions}

AMP use may be a valuable tool for assistant and postgraduate education, as it offers a more suitable view angle for ACL reconstruction.

\section{References}

1. Camp CL, Krych AJ, Stuart MJ, Regnier TD, Mills KM, Turner NS. Improving resident performance in knee arthroscopy: A prospective value assessment of simulators and cadaveric skills laboratories. J Bone Joint Surg Am 2016;98:220-225.

2. Butler A, Olson T, Koehler R, Nicandri G. Do the skills acquired by novice surgeons using anatomic dry models transfer effectively to the task of diagnostic knee arthroscopy performed on cadaveric specimens? J Bone Joint Surg Am 2013;95:e15.

3. Frank RM, Erickson B, Frank JM, et al. Utility of modern arthroscopic simulator training models. Arthroscopy 2014;30:121-133.

4. Cannon WD, Garrett WE Jr, Hunter RE, et al. Improving residency training in arthroscopic knee surgery with use of a virtual-reality simulator: A randomized blinded study. J Bone Joint Surg Am 2014;96:1798-1806.

5. Mabrey JD, Gillogly SD, Kasser JR, et al. Virtual reality simulation of arthroscopy of the knee. Arthroscopy 2002;18:1-7.

6. Martin RK, Gillis D, Leiter J, Shantz JS, MacDonald P. A porcine knee model is valid for use in the evaluation of arthroscopic skills: A pilot study. Clin Orthop Relat Res 2016;474:965-970.

7. Martin KD, Belmont PJ Jr, Schoenfeld AJ, Todd M, Cameron KL, Owens BD. Arthroscopic basic task performance in shoulder simulator model correlates with similar task performance in cadavers. J Bone Joint Surg Am $2011 ; 93:$ el27.

8. Henn RF III, Shah N, Warner JJ, Gomoll AH. Shoulder arthroscopy simulator training improves shoulder arthroscopy performance in a cadaveric model. Arthroscopy 2013;29:982-985. 
9. Martin KD, Cameron K, Belmont PJ Jr, Schoenfeld A, Owens BD. Shoulder arthroscopy simulator performance correlates with resident and shoulder arthroscopy experience. J Bone Joint Surg Am 2012;94:e160.

10. Martin KD, Patterson D, Phisitkul P, Cameron KL, Femino J, Amendola A. Ankle arthroscopy simulation improves basic skills, anatomic recognition, and proficiency during diagnostic examination of residents in training. Foot Ankle Int 2015;36:827-835.

11. Erturan G, Alvand A, Judge A, Pollard TC, Glyn-Jones S, Rees JL. Prior generic arthroscopic volume correlates with hip arthroscopic proficiency: a simulator study. J Bone Joint Surg Am 2018;100:e3.

12. Obdeijn M, Bavinck N, Mathoulin C, van der Horst C, Schijven M, Tuijthof G. Education in wrist arthroscopy: Past, present and future. Knee Surg Sports Traumatol Arthrosc 2015;23:1337-1345.

13. Yaacoub F, Hamam Y, Abche A. Computer-based training system for simulating wrist arthroscopy. 200821 st IEEE International Symposium on Computer-Based Medical Systems, Jyvaskyla, Finland, 2008; 421-423.

14. Angelo RL, Ryu RK, Pedowitz RA, et al. A proficiencybased progression training curriculum coupled with a model simulator results in the acquisition of a superior arthroscopic Bankart skill set. Arthroscopy 2015;31: 1854-1871.

15. Kohn D, Busche T, Carls J. Drill hole position in endoscopic anterior cruciate ligament reconstruction: Results of an advanced arthroscopy course. Knee Surg Sports Traumatol Arthrosc 1998;6:S13-S15.

16. Folse JR. Presidential address: surgical education-addressing the challenges of change. Surgery 1996; 120:575-579.

17. Collins A. Cognitive apprenticeship and instructional technology. In: Idol L, Jones BF, eds. Educational values and cognitive instruction: Implications for reform. Mahwah, NJ: Lawrence Erlbaum Associates, Inc., 1991;121-138.

18. Collins A, Brown JS, Holum A. Cognitive apprenticeship: Making thinking visible. Am J Educ 1991;15:6-11.

19. Collins A, Brown JS, Newman SE. Cognitive apprenticeship: Teaching the craft of reading, writing and mathematics. Thinking: The Journal of Philosophy for Children 1988;8:2-10.

20. Dennen VP, Burner KJ. The cognitive apprenticeship model in educational practice. Handbook Res Educ Commun Technol 2008;3:425-439.

21. Farmer JA Jr, Buckmaster A, LeGrand B. Cognitive apprenticeship: Implications for continuing professional education. New Dir Adult Cont Educ1992 1992:41-49.

22. Oranga $\mathrm{H}$, Nordberg E. The Delphi panel method for generating health information. Health Policy Plan 1993;8: 405-412.

23. Bruneau NN, Thorburn MA, Stevenson RM. Use of the Delphi panel method to assess expert perception of the accuracy of screening test systems for infectious pancreatic necrosis virus and infectious hematopoietic necrosis virus. J Aquat Anim Health 1999;1 1:139-147.

24. Jones J, Hunter D. Qualitative research: Consensus methods for medical and health services research. BMJ 1995:311:376-380.
25. Van Teijlingen E, Pitchforth E, Bishop C, Russell E. Delphi method and nominal group techniques in family planning and reproductive health research. J Fam Plann Reprod Health Care 2006;32:249-252.

26. Angelo RL, Ryu RK, Pedowitz RA, Gallagher AG. Metric development for an arthroscopic Bankart procedure: Assessment of face and content validity. Arthroscopy 2015;31:1430-1440.

27. Khalfayan EE, Sharkey PF, Alexander AH, Bruckner JD, Bynum EB. The relationship between tunnel placement and clinical results after anterior cruciate ligament reconstruction. Am J Sports Med 1996;24:335-341.

28. Sadoghi P, Kröpfl A, Jansson V, Müller PE, Pietschmann MF, Fischmeister MF. Impact of tibial and femoral tunnel position on clinical results after anterior cruciate ligament reconstruction. Arthroscopy 2011;27: 355-364.

29. Lee MC, Seong SC, Lee S, et al. Vertical femoral tunnel placement results in rotational knee laxity after anterior cruciate ligament reconstruction. Arthroscopy 2007;23: 771-778.

30. Biswal UK, Balaji G, Nema S, Poduval M, Menon J, Patro DK. Correlation of tunnel widening and tunnel positioning with short-term functional outcomes in single-bundle anterior cruciate ligament reconstruction using patellar tendon versus hamstring graft: A prospective study. Eur J Orthop Surg Traumatol 2016;26: 647-655.

31. Morgan JA, Dahm D, Levy B, Stuart MJ, Group MS. Femoral tunnel malposition in ACL revision reconstruction. J Knee Surg 2012;25:361-368.

32. Harner CD, Honkamp NJ, Ranawat AS. Anteromedial portal technique for creating the anterior cruciate ligament femoral tunnel. Arthroscopy 2008;24:113-115.

33. Pastrone A, Ferro A, Bruzzone M, et al. Anterior cruciate ligament reconstruction creating the femoral tunnel through the anteromedial portal. Surgical technique. Curr Rev Musculoskelet Med 201 1;4:52-56.

34. Lubowitz JH. Anteromedial portal technique for the anterior cruciate ligament femoral socket: Pitfalls and solutions. Arthroscopy 2009;25:95-101.

35. Asagumo H, Kimura M, Kobayashi Y, Taki M, Takagishi K. Anatomic reconstruction of the anterior cruciate ligament using double-bundle hamstring tendons: Surgical techniques, clinical outcomes, and complications. Arthroscopy 2007;23:602-609.

36. Cohen SB, Fu FH. Three-portal technique for anterior cruciate ligament reconstruction: Use of a central medial portal. Arthroscopy 2007;23:325.e1-325.e5.

37. Araujo PH, van Eck CF, Macalena JA, Fu FH. Advances in the three-portal technique for anatomical single-or double-bundle ACL reconstruction. Knee Surg Sports Traumatol Arthrosc $2011 ; 19: 1239-1242$.

38. Long W, Scott W. Anterior cruciate ligament injuries and reconstruction: Indications, principles and outcomes. Scott WN Insall e Scott Surg Knee 2012;5:371-384.

39. Sommer C, Friederich NF, Müller W. Improperly placed anterior cruciate ligament grafts: Correlation between radiological parameters and clinical results. Knee Surg Sports Traumatol Arthrosc 2000;8:207-213. 
40. Sven S, Maurice B, Hoeher J, Marc B. Variability of tunnel positioning in fluoroscopic-assisted ACL reconstruction. Knee Surg Sports Traumatol Arthrosc 2015;23: 2269-2277.

41. Siebold R, Fu FH. Assessment and augmentation of symptomatic anteromedial or posterolateral bundle tears of the anterior cruciate ligament. Arthroscopy 2008;24: 1289- 1298.

42. Ceponis PJ, Chan D, Boorman RS, Hutchison C, Mohtadi NG. A randomized pilot validation of educational measures in teaching shoulder arthroscopy to surgical residents. Can J Surg 2007;50:387. 